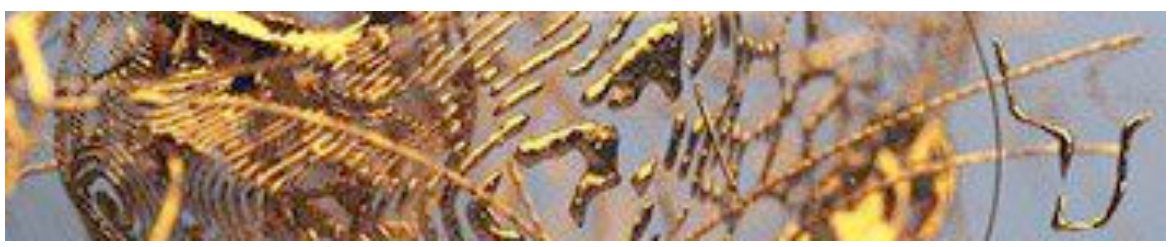

\title{
El uso de Twitter en las salas de redacción: redes sociales y newsmaking en la región sureste de Coahuila, México ${ }^{1}$
}

\section{Twitter usage in newsrooms: social media and newsmaking at Southeastern Coahuila, Mexico}

Pérez Salazar, Gabriel ${ }^{(*)}$

Universidad Nacional Autónoma de México - México

gabrielperezsalazar@gmail.com

Carabaza González, Julieta I. (**) $^{(*)}$

Universidad Nacional Autónoma de Coahuila -México jicarabazag@gmail.com

Romo Gutiérrez, Daniel O. ${ }^{(* * *)}$

Universidad Nacional Autónoma de Coahuila - México daniel.osvaldo.rg@gmail.com

${ }^{1}$ Este trabajo ha sido hecho con apoyo del Programa de Mejoramiento del Profesorado (PROMEP) y del Programa Integral de Fortalecimiento Institucional (PIFI), ambos de la Secretaría de Educación Pública, y forma parte de los resultados de investigación del Cuerpo Académico en Consolidación "Medios Masivos" de la Facultad de Ciencias de la Comunicación de la Universidad Autónoma de Coahuila. 


\section{Resumen}

El uso de las herramientas para la administración de redes sociales se está generalizando en algunas de las rutinas laborales de los trabajadores en los medios de comunicación, particularmente en los de carácter noticioso. Con base en una encuesta en línea y un conjunto de entrevistas, se presenta un panorama general del uso de Internet y de las redes sociales en una muestra no probabilística de reporteros, editores y dueños de medios de la región sureste de Coahuila; así como el tratamiento que un subgrupo de ellos le da a Twitter como fuente de información. Los principales resultados muestran que Internet es el medio al que dedican un mayor tiempo de consumo, y que Twitter es empleado como una herramienta que permite a los trabajadores de los medios, seguir asuntos de potencial interés periodístico, una vez que pasan por los procedimientos habituales de verificación de fuentes.

Palabras clave: redes sociales, Internet, periodismo convergente, gatekeeper, Twitter.

\section{Abstract}

The use of social network sites has increased in the newsmaking routines of media workers and journalists. Based on an online survey and a series of interviews, this paper shows some general trends about Internet usage and the way that some editors are handling Twitter as information source, in Southeastern Coahuila, México. Main results indicates that Internet is the most used media, and following Twitter allows them to keep track of potential breaking news, all sources verification procedures considered.

Keywords: Social Networks Sites (SNS), Internet, Digital Journalists, Gatekeeper, Twitter.

Mediaciones Sociales, № 12, 2013, pp. 108-128. ISSN electrónico: 1989-0494.

DOI: http://dx.doi.org/10.5209/rev_MESO.2013.n12.45265 


\section{INTRODUCCIÓN}

El uso de las tecnologías de la información y la comunicación (TIC) ha ido extendiéndose en muchas áreas de los entornos productivos contemporáneos. A pesar de que en México prevalece una profunda brecha digital2, Internet en general, y las herramientas para la administración de redes sociales en línea, en particular, están cada vez más presentes en empresas, universidades, oficinas de gobierno, así como entre los trabajadores en los medios de comunicación.

Twitter es una de estas plataformas que está ganando usuarios en México $^{3}$, y que, por sus lógicas operativas, resulta muy cercana a las actividades que realizan los profesionales de los medios informativos, especialmente entre reporteros, editores y jefes de sección. Esta plataforma de microblogging permite una rápida difusión de información de toda índole entre sus usuarios, destacando la de tipo noticioso, a pesar del carácter no profesional de muchos de sus lecto-emisores"

Adentrarse en los usos sociales de esta tecnología, en especial a partir de su incorporación dentro de lo que se conoce como la sociología del newsmaking; permite actualizar una de las corrientes teóricas más ampliamente abordadas desde el campo académico de la Comunicación. Su condición como herramienta info-comunicacional, a la vez que plataforma de interacción social mediada por computadora, con flujos multidireccionales y relativamente descentralizados; tiene el potencial de influir de manera importante en el tratamiento de fuentes y de sondeo de la esfera noticiosa. Asimismo, este tipo de aplicaciones introduce tensiones entre dichos profesionales de la noticia al enfrentar la inmediatez y la proximidad mediada, contra algunos de los pilares fundamentales del periodismo, en especial en

2 Internet World Stats (2012) reporta una tasa de penetración de Internet en México de sólo el $36.9 \%$

3 Octavio Islas, Director del Proyecto Internet del Instituto Tecnológico y de Estudios Superiores de Monterrey, Campus Ciudad de México, calcula un estimado de 8.5 millones de usuarios de Twitter en México (Islas, 2012).

4 Empleamos este término para resaltar el sentido que tienen las acciones que los usuarios de este tipo de sistemas llevan a cabo, y que van más allá de la mera lectura. Esta figura parte de al menos dos antecedentes: la noción del emirec propuesta por Cloutier (1973) y los wreaders de Landow (1994). Este término supera ampliamente el de prosumers, planteado frecuentemente desde una visión fundamentalmente instrumental y comercial de Internet en general, y la Web 2.0 en particular.

Mediaciones Sociales, № 12, 2013, pp. 108-128. ISSN electrónico: 1989-0494. DOI: http://dx.doi.org/10.5209/rev_MESO.2013.n12.45265 
lo que tiene que ver con la validación de la información, la veracidad y la verificación de fuentes.

El presente trabajo presenta un estudio exploratorio sobre el uso de Internet y de las redes sociales en la región sureste de Coahuila, México, enfocándose en la manera en que los trabajadores en los medios de comunicación emplean dichas tecnologías. La credibilidad de la información que circula en Twitter, así como su presencia en las salas de redacción de los tres periódicos con mayor circulación en Saltillo, son algunos aspectos específicos abordados.

\section{ANTECEDENTES Y MARCO TEÓRICO-CONCEPTUAL}

\subsection{Estado del arte}

El uso de las tecnologías de la información y la comunicación por parte de los periodistas y profesionales de la información es un tema que ha sido tratado por muy diversos autores. Algunos de los antecedentes más destacados se deben al trabajo de Jenkins (2006), quien habla de la manera en que los usuarios de Internet se enfrentan a los contenidos de este medio, de forma distinta a los de los medios tradicionales; en particular en relación con las noticias. Como este autor propone, en Internet los usuarios tienen la posibilidad de llevar a cabos procesos de búsqueda y selección de información noticiosa de manera activa, con base en sus intereses y necesidades.

Como Meneses (2011) plantea, la llegada de la convergencia tecnológica a las salas de redacción de las empresas noticiosas en México ha traído consigo numerosas transformaciones en esta actividad profesional, incluso desde antes de la generalización en algunos sectores, del uso de las herramientas para la administración de redes sociales en línea, cuando se discutía en torno al papel de blogs y foros en el contexto noticioso. De forma específica, la introducción de dichas herramientas en las salas de redacción y su empleo por parte de editores y periodistas ha sido objeto de análisis en trabajos como los que mencionaremos enseguida.

A partir de una reflexión sobre el uso de MySpace y Facebook, Spencer (2007) destaca el papel de estas herramientas como fuente de información para los periodistas. A través de esta mediación, sostiene este autor, es posible tener acceso a sujetos que pueden encontrarse en medio de

Mediaciones Sociales, № 12, 2013, pp. 108-128. ISSN electrónico: 1989-0494.

DOI: http://dx.doi.org/10.5209/rev_MESO.2013.n12.45265 
eventos noticiosos de talla internacional, como guerras y catástrofes naturales. Desde un punto de vista similar, Overholser (2009) coincide con este autor en relación con el potencial de los llamados social media para reconfigurar algunas prácticas periodísticas, enfatizando, no obstante, la necesidad de mantener los valores fundamentales de un verdadero periodista: una actitud crítica y la verificación de las fuentes.

Lariscya et al (2009) presentan un estudio relativo a la frecuencia con que un grupo de 200 periodistas del área de negocios en los Estados Unidos emplean sitios como Facebook y YouTube como fuentes de información. Aunque estos autores encuentran que los integrantes de la muestra no recurren de forma sistemática a estos sitios para elaborar sus notas, sugieren tomar en cuenta estas plataformas para un futuro en el que su uso sea más generalizado.

Refiriéndose de manera específica a Twitter, Hermida (2010) propone que el uso de esta herramienta está dando lugar a lo que llama un ambiente periodístico ${ }^{5}$, a partir de los flujos de información que generan sus usuarios. En concordancia con lo propuesto por Overholster, este flujo, dice Hermida, da a las noticias una dimensión de experiencia social, en la que es necesario fortalecer competencias como la selección y el filtrado de información. El ambiente periodístico planteado por este autor de ninguna manera implica que usuarios sin formación en periodismo estén ejerciendo dicha actividad, sino que, en cercanía con la noción de la inteligencia colectiva de Lèvy (1997), el conjunto de todos los tweets puede dar lugar a procesos colectivos en los que la información noticiosa permea transversalmente dichos entornos virtuales.

Aunque autores como Overholster (2009) y Spencer (2007) han expresado reflexiones en torno a si los medios sociales pueden afectar de alguna manera la esencia del quehacer periodístico, justo por este uso descrito por Hermida; el planteamiento de Gabrielle Levy (2010) se centra en la idea de que, para aquellos que ejercen el periodismo, herramientas como Twitter y Facebook están tomando una importancia creciente en sus rutinas profesionales, a pesar de que usuarios sin un entrenamiento específico para ello, se estén convirtiendo en emisores de información noticiosa. Desde esta perspectiva, la cuestión no se centra tanto en lo que emiten los

\footnotetext{
5 Ambient journalism, en el original.
} 
usuarios comunes y corrientes, sino en la forma en que los periodistas profesionales se apropian de dichas tecnologías.

Las conclusiones de Levy (2010) coinciden con los hallazgos de Jürgens, Jungherr y Schoen (2011), en relación con estos procesos de incorporación de Twitter entre los periodistas. Con base en una observación realizada durante las elecciones presidenciales de 2009 en Alemania, estos autores encuentran que muchos periodistas y líderes de opinión, evaluaron la aceptación de los candidatos, a partir de los comentarios emitidos a través de Twitter. De esta manera, los medios tradicionales operaron como una suerte de caja de resonancia del clima de opinión generado desde esta plataforma. Retomando la noción funcionalista de los guardabarreras (gatekeepers) y combinándola con la idea de los nodos concentradores ${ }^{6}$ desarrollada por Watts y Strogatz (en Jürgens, Jungherr y Schoen, 2011); estos investigadores proponen que los periodistas alemanes llevaron a cabo una selección de datos e información originados en Twitter, para ser retomados en otros medios con mayor alcance, como la prensa, radio y televisión.

\subsection{Marco de referencia}

Como es posible observar, una parte de los antecedentes consultados resalta el papel que los profesionales de la noticia tienen en la selección y filtrado de la información, en cercanía con lo señalado por la Teoría del Guardabarreras (gatekeeper), misma que revisaremos brevemente a continuación.

Wolf (2005) señala que la noción inicial del guardabarreras corresponde a Kurt Lewin, quien en 1947 publicó un trabajo sobre las dinámicas de interacción al interior de los grupos sociales. Al observar que algunos sujetos actuaban como filtros en la información que se transmite al interior de un sistema social, este autor caracterizó a los guardabarreras como aquellos individuos o grupos que tienen el "poder de decidir si dejar pasar o bloquear la información” (Lewin en Wolf, 2005: 205).

Maigret (2005) apunta que fue White el primero en aplicar este concepto al trabajo de los periodistas. Con base en una investigación realizada por este autor, se concluye que la experiencia personal (y no tanto los su-

\footnotetext{
6 Dentro de la teoría de las redes sociales, los nodos concentradores son puntos capaces de establecer puentes entre distintos conjuntos de elementos de la red, y dar lugar a flujos de información más amplios y veloces, de lo que la misma teoría predice.
} 
puestos criterios de objetividad), explica buena parte de la selección de noticias hecha por los editores y reporteros. Esto, aunado a las rutinas burocráticas (sic), llevan a que en las empresas noticiosas se dé prioridad a eventos negativos o dramáticos, con base en fuentes oficiales.

Como Shoemaker y Reese (1994) señalan, de todas las posibles notas que llegan a la sala de redacción de un periódico, sólo un pequeño porcentaje (menos del $10 \%$ ), es finalmente publicado, a partir de estos criterios de selección que han sido señalados. Si, como Hermida (2010) sostiene, Twitter da lugar a un ambiente periodístico, ciertamente no toda la información que circula por esta plataforma es retomada por los medios noticiosos. Las razones, además de los criterios que ya han sido mencionados, tienen que ver también con la lógica de este medio de difusión de información. Como Jürgens, Jungherr y Schoen (2011) señalan, cualquier usuario es capaz de generar flujos de información, sin que necesariamente haya un tratamiento profesional de la misma; lo cual implica una consideración importante durante los procesos de edición noticiosa.

Con base en lo anteriormente planteado, es que se llevó a cabo un trabajo de campo aplicado en la región sureste de Coahuila, a partir de dos acercamientos: una encuesta que da una idea general sobre el uso de Internet y redes sociales en este contexto geográfico y, en segundo lugar, un conjunto de entrevistas a editores de los tres diarios con mayor circulación en la ciudad de Saltillo, Coahuila (México).

\section{DESCRIPCIÓN METODOLÓGICA}

Como ha sido adelantado, se emplearon dos estrategias para el levantamiento de datos: 1) una encuesta aplicada a usuarios de Internet residentes en la región sureste de Coahuila y 2) un conjunto de entrevistas hechas a editores de los periódicos Vanguardia, Zócalo Saltillo y El Diario de Coahuila.

\subsection{Detalles metodológicos de la encuesta}

Se aplicó una encuesta en línea a una muestra no probabilística de residentes en la región sureste de Coahuila (figura 1), mayores de edad y usuarios de Internet. Los participantes fueron voluntarios reclutados a través de 2,694 invitaciones hechas por correo electrónico, por medio del sistema SurveyMonkey, a partir de una base de datos elaborada con los 
contactos de los miembros del Cuerpo Académico en Consolidación al que pertenecen los autores de este trabajo. El periodo de invitación y recepción de encuestas comprendió del 9 de enero al 9 de febrero de 2012. Al cierre, se obtuvieron 325 registros en el sistema, lo que da un índice de respuesta del $12.06 \%$. Cabe señalar que este porcentaje es menor al reportado por Kongsved et al (2007), quienes compararon las tasas de respuesta de cuestionarios aplicados de forma presencial $(73.2 \%)$, contra los administrados a través de Internet (17.9\%).

Figura 1. Región sureste de Coahuila

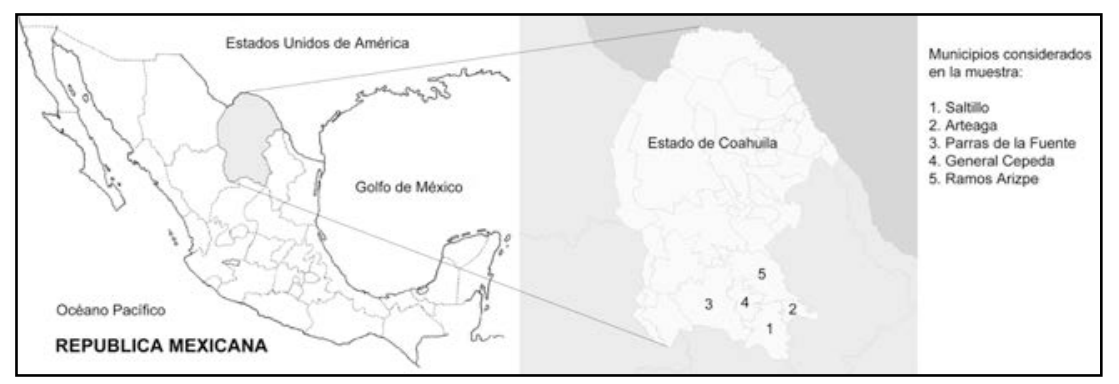

Fuente: Elaboración propia

De los 325 cuestionarios con respuestas registradas, 47 personas vivían en otras localidades fuera de la región sureste de Coahuila, por lo cual fueron eliminados. Otros 99 registros fueron suprimidos debido a que sólo contestaron los ítems ubicados en la primera página del cuestionario y el resto fue dejado en blanco. Para el presente trabajo sólo se consideran los 179 cuestionarios que fueron contestados de manera completa por usuarios que declararon su residencia en dicha región.

Aunque se trataba de un cuestionario diseñado para medir diversas variables ${ }^{7}$, en el presente trabajo presentamos sólo aquellos indicadores que tienen una relación directa con los procesos de uso y apropiación de redes sociales, en general, y de Twitter, en particular, por parte de trabajadores en los medios masivos de comunicación de la muestra.

\footnotetext{
7 Entre las que destacan la interacción mediada por las TICs, así como patrones de uso y apropiación de dichas tecnologías.
} 


\subsection{Detalles metodológicos de las entrevistas}

Se realizó un conjunto de entrevistas semi-estructuradas a los editores de las secciones locales de los tres periódicos con mayor circulación en Saltillo, Coahuila (Vanguardia, Zócalo Saltillo y El Diario de Coahuila), en la que, a partir de 14 ítems, se buscó establecer la frecuencia con la que estos trabajadores en los medios impresos que actúan como guardabarreras, retoman la información que reciben vía Twitter para su posible publicación ${ }^{8}$.

\section{Resultados}

La encuesta realizada permite tener una apreciación general del uso que un grupo no probabilístico de usuarios de Internet, hace de las herramientas info-comunicativas disponibles en esta red, con énfasis en los servicios que permiten administrar redes sociales en línea. Se presentan los resultados de este acercamiento metodológico en primer lugar, para mostrar posteriormente el resultado de las entrevistas semi-estructuradas.

De los 179 cuestionarios válidos, para el presente trabajo se seleccionaron aquellos usuarios que dijeron trabajar en algún medio de comunicación. El total de las actividades declaradas por los participantes en la encuesta se muestra en la tabla 1.

Tabla 1. Campo de actividad laboral

\begin{tabular}{lcc} 
& $f$ & $\%$ \\
\hline Sector educativo & 78 & 44 \\
Medios de comunicación & 33 & 18 \\
Sector público & 25 & 14 \\
Sector empresarial & 21 & 12 \\
Sector industrial & 12 & 7 \\
Comercio & 5 & 3 \\
Sector salud & 3 & 2 \\
Total & $177^{9}$ & 100 \\
\hline
\end{tabular}

Fuente: elaboración propia

8 Estas entrevistas fueron realizadas por Daniel Osvaldo Romo Gutiérrez, como parte de su trabajo de tesis de licenciatura.

9 De los 179 cuestionarios válidos, dos personas dijeron estar pensionadas por jubilación, lo que deja 177 respuesta válidas para esta variable.

Mediaciones Sociales, № 12, 2013, pp. 108-128. ISSN electrónico: 1989-0494.

DOI: http://dx.doi.org/10.5209/rev_MESO.2013.n12.45265 
Como es posible observar, el subgrupo considerado en este trabajo es el segundo más numeroso dentro de la muestra y está integrado por 33 personas (18\%). Los resultados que se muestran en primer lugar tienen que ver con un conjunto de datos socio demográficos generales, contrastados en algunos casos, con la muestra en general.

En la tabla 2 se presenta la composición de los trabajadores en medios, por sexo; en la que es evidente que este subsector no representa proporcionalmente esta variable en relación con la muestra.

Tabla 2. Participantes en la encuesta, por sexo

\begin{tabular}{lcc}
\hline & Muestra (\%) & Trabajadores en medios (\%) \\
\hline Hombres & 58.0 & 66.7 \\
Mujeres & 42.0 & 33.3 \\
Total & 100.0 & 100.0 \\
\hline
\end{tabular}

Fuente: elaboración propia

La distribución de edades de los trabajadores en medios se presenta en la tabla 3, y en ella destaca que el rango más frecuente se encuentra entre los 38 y los 42 años. El promedio de edad dentro de este grupo es de 37.39 años, con una desviación estándar de 13.27 años.

Tabla 3. Grupos de edades Trabajadores en medios (años cumplidos)

\begin{tabular}{lcc}
\hline Grupos de edad & $f$ & $\%$ \\
\hline 18 a 22 & 5 & 15.15 \\
23 a 27 & 5 & 15.15 \\
28 a 32 & 5 & 15.15 \\
33 a 37 & 3 & 9.09 \\
38 a 42 & 7 & 21.21 \\
43 a 47 & 2 & 6.06 \\
48 a 52 & 2 & 6.06 \\
53 y mayores & 4 & 12.12 \\
Total & 33 & 100.0 \\
\hline
\end{tabular}

Fuente: elaboración propia

En lo relativo al nivel de estudios, dentro de los trabajadores en medios es posible apreciar que la mayor parte de los que componen este subgrupo, cuentan con la licenciatura terminada, pero pocos con algún posgrado. 
Gabriel Pérez Salazar, Julieta I. Carabaza González y Daniel O. Romo Gutiérrez

Tabla 4. Participantes en encuesta, por nivel educativo (porcentajes)

\begin{tabular}{lcc}
\hline & Muestra (\%) & $\begin{array}{c}\text { Trabajadores en } \\
\text { medios (\%) }\end{array}$ \\
\hline Bachillerato completo & 6.0 & 12.1 \\
Licenciatura incompleta & 2.0 & 18.2 \\
Licenciatura completa & 32.0 & 60.6 \\
Maestría incompleta & 6.0 & 3.0 \\
Maestría completa & 27.0 & 3.0 \\
Doctorado incompleto & 4.0 & - \\
Doctorado completo & 22.0 & 3.0 \\
Carrera técnica incompleta & 1.0 & - \\
Total & 100.0 & 100.0 \\
\hline
\end{tabular}

Fuente: elaboración propia

De las 33 personas que dijeron ser trabajadores en los medios, sólo 28 especificaron su función. Como es posible apreciar en la tabla 5 , aunque la mayor proporción corresponde a los reporteros (57\%), hay tres dueños de medios dentro de este sub-grupo.

Tabla 5. Función que desempeñan los trabajadores en medios

\begin{tabular}{lcc}
\hline & $f$ & $\%$ \\
\hline Empleado especializado (reportero) & 16 & 57 \\
Jefe de área & 4 & 14 \\
Directivo & 4 & 14 \\
Dueño de medio de comunicación & 3 & 11 \\
Servicios de apoyo en forma independiente & 1 & 4 \\
Total & 28 & 100 \\
\hline
\end{tabular}

Fuente: elaboración propia

Como se puede observar en la tabla 6 , de los 28 trabajadores en medios participantes en la encuesta que especificaron su función, la totalidad de los reporteros cuenta al menos con estudios iniciales de licenciatura, y el $66 \%$ de ellos, tienen este nivel concluido. En lo que respecta a los dueños de los medios, de los tres casos reportados, uno tiene licenciatura incompleta, uno maestría incompleta y el otro, maestría terminada. 
Gabriel Pérez Salazar, Julieta I. Carabaza González y Daniel O. Romo Gutiérrez

Tabla 6. Nivel estudios contra función que desempeñan trabajadores en los medios ${ }^{10}$

\begin{tabular}{|c|c|c|c|c|c|c|}
\hline & $\begin{array}{l}\text { Prepara- } \\
\text { toria } \\
\text { completa }\end{array}$ & $\begin{array}{l}\text { Licencia- } \\
\text { tura } \\
\text { incom- } \\
\text { pleta }\end{array}$ & $\begin{array}{l}\text { Licencia- } \\
\text { tura } \\
\text { completa }\end{array}$ & $\begin{array}{c}\text { Maes- } \\
\text { tría } \\
\text { incom- } \\
\text { pleta }\end{array}$ & $\begin{array}{c}\text { Maes- } \\
\text { tría } \\
\text { com- } \\
\text { pleta }\end{array}$ & $\begin{array}{l}\text { To- } \\
\text { tal }\end{array}$ \\
\hline $\begin{array}{l}\text { Servicios de apoyo en forma } \\
\text { independiente }\end{array}$ & 1 & & & & & 1 \\
\hline $\begin{array}{l}\text { Dueño de medio de comunica- } \\
\text { ción }\end{array}$ & & 1 & & 1 & 1 & 3 \\
\hline $\begin{array}{l}\text { Empleado especializado (repor- } \\
\text { tero) }\end{array}$ & & 4 & 12 & & & 16 \\
\hline Jefe de área & 1 & & 3 & & & 4 \\
\hline Directivo & & 1 & 3 & & & 4 \\
\hline Total & 2 & 6 & 18 & 1 & 1 & 28 \\
\hline
\end{tabular}

Fuente: elaboración propia

Cuando se compara el consumo de medios (tabla 7) que hacen los 33 trabajadores en medios de la muestra, contra el consumo específico que realizan los reporteros y los dueños de los medios, es posible observar notables diferencias entre ellos. Aunque en todos los casos Internet es el medio al que dedican mayor tiempo, mientras que los reporteros en promedio dicen ver casi tres cuartos de hora de TV abierta (44.2 minutos), ninguno de los dueños de medios hace este tipo de consumo televisivo. En cuanto a la radio, los reporteros acuden a este medio casi 2.8 veces más que los dueños de los medios. En todos ellos, el medio que menos consumen son las revistas.

Tabla 7. Consumo de medios (minutos al día)

\begin{tabular}{|c|c|c|c|c|c|c|}
\hline Función & $\begin{array}{l}\text { Nave- } \\
\text { gando en } \\
\text { Internet }\end{array}$ & $\begin{array}{c}\text { Vien- } \\
\text { do } \\
\text { TV } \\
\text { por } \\
\text { cable }\end{array}$ & $\begin{array}{l}\text { Escu- } \\
\text { chando } \\
\text { radio }\end{array}$ & $\begin{array}{l}\text { Leyen- } \\
\text { do } \\
\text { periódi- } \\
\text { cos }\end{array}$ & $\begin{array}{c}\text { Vien- } \\
\text { do } \\
\text { TV } \\
\text { abier- } \\
\text { ta }\end{array}$ & $\begin{array}{l}\text { Le- } \\
\text { yendo } \\
\text { revis- } \\
\text { tas }\end{array}$ \\
\hline $\begin{array}{l}\text { Trabajadores en medios (33 } \\
\text { respuestas) }\end{array}$ & 220.3 & 89.6 & 90.0 & 38.6 & 35.7 & 16.5 \\
\hline Reporteros (16 respuestas) & 240.0 & 115.1 & 106.9 & 35.6 & 44.2 & 12.4 \\
\hline Dueños de medios (3 respuestas) & 373.3 & 130.0 & 38.3 & 43.3 & 0.0 & 23.3 \\
\hline
\end{tabular}

Fuente: elaboración propia

${ }^{10}$ En esta tabla no aparecen los participantes en la encuesta que dijeron tener doctorado, porque en sus registros no declararon su función específica. Este cruce de variables está hecho sobre una base de 28 respuestas efectivas para la variable función, mientras que para la variable nivel de estudios, fueron 33 respuestas.

Mediaciones Sociales, № 12, 2013, pp. 108-128. ISSN electrónico: 1989-0494.

DOI: http://dx.doi.org/10.5209/rev_MESO.2013.n12.45265 
Al indagar sobre las actividades que realizan en Internet los trabajadores en medios de la muestra (tabla 8 ), en los cinco primeros lugares se encuentran el uso del correo electrónico, la navegación por la WWW, el empleo de buscadores, el uso de alguna herramienta para la administración de redes sociales en línea y el chat. A pesar del reducido tamaño de la muestra que corresponde a dueños de medios, es posible observar algunas diferencias notables en relación con el grupo de reporteros, especialmente en lo que tiene que ver con compras en línea, descarga de música y realización de trámites de gobierno; actividades realizadas con mayor frecuencia entre éstos primeros. Por el contrario, los reporteros presentan una mayor frecuencia sobre los dueños de los medios en actividades como leer blogs de otras personas, jugar en línea y recibir educación por Internet.

Tabla 8. Actividades que realizan en Internet los trabajadores en medios (porcentajes) ${ }^{11}$

\begin{tabular}{|c|c|c|c|}
\hline $\begin{array}{l}\text { Actividades que realizan en In- } \\
\text { ternet }\end{array}$ & $\begin{array}{l}\text { Trabajadores en } \\
\text { los medios }\end{array}$ & Reporteros & $\begin{array}{l}\text { Dueños de me- } \\
\quad \text { dios }\end{array}$ \\
\hline $\begin{array}{l}\text { Enviar o recibir correo electróni- } \\
\text { co }\end{array}$ & $100.0 \%$ & $100.0 \%$ & $100.0 \%$ \\
\hline Navegar por WWW & $97.0 \%$ & $93.8 \%$ & $100.0 \%$ \\
\hline $\begin{array}{l}\text { Buscar información de cualquier } \\
\text { tipo (uso de buscadores) }\end{array}$ & $97.0 \%$ & $93.8 \%$ & $100.0 \%$ \\
\hline $\begin{array}{l}\text { Entrar a alguna red social como } \\
\text { Facebook, MySpace, Hi-5 o Twit- } \\
\text { ter }\end{array}$ & $90.9 \%$ & $93.8 \%$ & $100.0 \%$ \\
\hline Chatear/Messenger & $75.8 \%$ & $81.3 \%$ & $100.0 \%$ \\
\hline $\begin{array}{l}\text { Ver en línea videos, películas o } \\
\text { series de televisión (sin descar- } \\
\text { garlas) }\end{array}$ & $72.7 \%$ & $62.5 \%$ & $100.0 \%$ \\
\hline Leer los blogs de otras personas & $63.6 \%$ & $75.0 \%$ & $66.7 \%$ \\
\hline $\begin{array}{l}\text { Escuchar música en línea (sin } \\
\text { descargarla) }\end{array}$ & $60.6 \%$ & $56.3 \%$ & $100.0 \%$ \\
\hline Descargar música & $54.5 \%$ & $62.5 \%$ & $100.0 \%$ \\
\hline $\begin{array}{l}\text { Videollamadas/llamadas de voz } \\
\text { (Skype, Gtalk, etc.) }\end{array}$ & $54.5 \%$ & $50.0 \%$ & $66.7 \%$ \\
\hline $\begin{array}{l}\text { Dejar comentarios en los blogs de } \\
\text { otras personas }\end{array}$ & $45.5 \%$ & $56.3 \%$ & $33.3 \%$ \\
\hline
\end{tabular}

Mediaciones Sociales, № 12, 2013, pp. 108-128. ISSN electrónico: 1989-0494. DOI: http://dx.doi.org/10.5209/rev_MESO.2013.n12.45265 
Gabriel Pérez Salazar, Julieta I. Carabaza González y Daniel O. Romo Gutiérrez

\begin{tabular}{|c|c|c|c|}
\hline $\begin{array}{l}\text { Colocar en línea material de su } \\
\text { propia creación como fotos o } \\
\text { videos }\end{array}$ & $42.4 \%$ & $50.0 \%$ & $33.3 \%$ \\
\hline Comprar en línea & $42.4 \%$ & $31.3 \%$ & $100.0 \%$ \\
\hline Trabajo colaborativo en línea & $39.4 \%$ & $25.0 \%$ & $33.3 \%$ \\
\hline Jugar en línea & $36.4 \%$ & $43.8 \%$ & $33.3 \%$ \\
\hline $\begin{array}{l}\text { Descargar videos, películas o } \\
\text { series de televisión }\end{array}$ & $30.3 \%$ & $25.0 \%$ & $66.7 \%$ \\
\hline Utilizar banca en línea & $30.3 \%$ & $18.8 \%$ & $66.7 \%$ \\
\hline Trámites de gobierno en línea & $30.3 \%$ & $18.8 \%$ & $100.0 \%$ \\
\hline $\begin{array}{l}\text { Dar de alta o actualizar su propio } \\
\text { blog }\end{array}$ & $27.3 \%$ & $43.8 \%$ & $66.7 \%$ \\
\hline Recibir educación en línea & $21.2 \%$ & $31.3 \%$ & $0.0 \%$ \\
\hline Declaración de impuestos en línea & $12.1 \%$ & $0.0 \%$ & $33.3 \%$ \\
\hline $\begin{array}{l}\text { Participar en mundos virtuales } \\
\text { como Second Life }\end{array}$ & $0.0 \%$ & $0.0 \%$ & $0.0 \%$ \\
\hline
\end{tabular}

Fuente: elaboración propia

En lo concerniente al uso de las herramientas para la administración de redes sociales en línea, de los 33 trabajadores en los medios presentes en la muestra, el 93.9\% (31 casos) dicen tener cuenta en alguna de estas plataformas. Como es posible apreciar en la tabla 9 , los dos servicios de este tipo más frecuentemente usados son Facebook y Twitter.

Tabla 9. Penetración de herramientas para la administración de redes sociales en línea, en trabajadores en medios de la muestra ${ }^{12}$

\begin{tabular}{lcc}
\hline & $f$ & $\%$ \\
\hline Facebook & 31 & $93.9 \%$ \\
Twitter & 28 & $84.8 \%$ \\
LinkedIn & 9 & $27.3 \%$ \\
Otras (Google+, Tagged, Sonic) & 5 & $15.2 \%$ \\
Hi-5 & 4 & $12.1 \%$ \\
MySpace & 1 & $3.0 \%$ \\
\hline
\end{tabular}

Fuente: elaboración propia

${ }^{12}$ La suma del total de porcentajes supera el $100 \%$ porque era un ítem que admitía múltiples respuestas.

Mediaciones Sociales, № 12, 2013, pp. 108-128. ISSN electrónico: 1989-0494. DOI: http://dx.doi.org/10.5209/rev_MESO.2013.n12.45265 
Los resultados relativos a las frecuencias con que son abordados los temas que se detallan en la tabla 10, dan cuenta de la importancia que tienen estas plataformas sobre asuntos que se asocian con cuatro aspectos en concreto: los que se refieren a sucesos noticiosos (entre los que pueden incluirse los relativos a seguridad pública), aquellos relacionados con el trabajo, y los relativos a la familia.

Tabla 10. Temas abordados en Facebook y Twitter por trabajadores en medios

\begin{tabular}{|c|c|c|c|c|c|c|c|}
\hline & \multicolumn{3}{|c|}{ Facebook } & \multicolumn{3}{|c|}{ Twitter } & \multirow[b]{2}{*}{$\begin{array}{l}\text { Total } \\
\text { por } \\
\text { tema } \\
(f)\end{array}$} \\
\hline & $\begin{array}{c}\text { Primer } \\
\text { tema } \\
\text { más } \\
\text { frecuente } \\
(f)\end{array}$ & $\begin{array}{c}\text { Segundo } \\
\text { tema } \\
\text { más } \\
\text { frecuente } \\
(f)\end{array}$ & $\begin{array}{c}\text { Tercer } \\
\text { tema } \\
\text { más } \\
\text { frecuente } \\
(f)\end{array}$ & $\begin{array}{c}\text { Temas } \\
\text { seguidos } \\
\text { de mane- } \\
\text { ra cons- } \\
\text { tante }(f)\end{array}$ & $\begin{array}{c}\text { Temas } \\
\text { seguidos } \\
\text { de ma- } \\
\text { nera } \\
\text { ocasional } \\
(f) \\
\end{array}$ & $\begin{array}{c}\text { Temas } \\
\text { sobre } \\
\text { los } \\
\text { que se } \\
\text { escribe } \\
(f) \\
\end{array}$ & \\
\hline $\begin{array}{l}\text { Noticias y temas } \\
\text { de actualidad }\end{array}$ & 2 & 11 & 9 & 16 & 4 & 5 & 47 \\
\hline $\begin{array}{l}\text { Asuntos de traba- } \\
\text { jo/escuela }\end{array}$ & 21 & & & 12 & 4 & 3 & 40 \\
\hline Seguridad pública & & 2 & 3 & 14 & 4 & 4 & 27 \\
\hline Asuntos familiares & 2 & 9 & & 4 & 7 & 1 & 23 \\
\hline $\begin{array}{l}\text { Tiempo libre, } \\
\text { humor, películas, } \\
\text { libros, etc. }\end{array}$ & & & 4 & 4 & 9 & $\mathrm{O}$ & 17 \\
\hline $\begin{array}{l}\text { Temas relaciona- } \\
\text { dos con relaciones } \\
\text { personales o sen- } \\
\text { timentales }\end{array}$ & & 1 & 4 & 2 & 4 & 4 & 15 \\
\hline $\begin{array}{l}\text { Asuntos sobre el } \\
\text { medio ambiente }\end{array}$ & & & 1 & 3 & 8 & 2 & 14 \\
\hline $\begin{array}{l}\text { Asuntos relacio- } \\
\text { nados con el go- } \\
\text { bierno }\end{array}$ & & & 1 & 4 & 6 & 2 & 13 \\
\hline $\begin{array}{l}\text { Total de respues- } \\
\text { tas registradas en } \\
\text { cada caso }\end{array}$ & 25 & 23 & 22 & 59 & 46 & 21 & 196 \\
\hline
\end{tabular}

Fuente: elaboración propia

En lo relativo a los resultados de las entrevistas, se encontró que las redes sociales en general y Twitter en particular son medios empleados habitualmente por los editores de las secciones locales de los 
tres periódicos considerados en la muestra13, en coincidencia con lo observado a través de la encuesta. No obstante este relativamente frecuente uso de Twitter, los tres participantes en el estudio señalaron emplearlo en pocas ocasiones como fuente directa para la generación de notas. De forma específica, el editor de la sección local de El Diario de Coahuila expresó que en su opinión, la mayor parte de la información que es recibida por Twitter es "falsa, con numerosos rumores y deformaciones de los hechos".

La credibilidad de la información que circula por Twitter es señalada como un problema, de forma recurrente por los tres editores entrevistados. En una escala tipo Lickert de cero a diez14, el promedio de las respuestas para esta variable se ubicó en cinco. Las prácticas periodísticas habituales, como la confirmación de fuentes, siguen siendo la norma. Para verificar algún dato de interés periodístico publicado en Twitter, la mayor parte de los editores (dos de tres) señalaron que envían un reportero al lugar de los hechos, si la nota lo justifica por su importancia. En este sentido, los criterios de noticiabilidad señalados por los entrevistados son la trascendencia, la actualidad y la proximidad de la información.

Las fuentes a las que recurren los editores de estos tres periódicos a través de Twitter son: fuentes formales (cuentas de funcionarios y dependencias oficiales), colegas periodistas y fuentes informales (usuarios testigos de algún hecho, pero no protagonistas).

\section{DISCUSIÓN Y CONCLUSIONES}

A partir de los resultados mostrados, es posible resaltar en primer lugar, la importancia que tienen las TICs para los trabajadores en los medios que participaron en las aproximaciones metodológicas que fueron aplicadas. Uno de los primeros hallazgos a destacar es que el consumo de Internet supera el de cualquier otro medio de comunicación de masas, dentro de la

\footnotetext{
${ }^{13}$ Los participantes en las entrevistas dijeron tener un máximo de dos años de haber empezado a usar Twitter.

${ }^{14}$ Siendo cero una credibilidad nula y diez una credibilidad total.
} 
muestra participante en la encuesta. Este hecho da cuenta de un intenso uso de los entornos virtuales, mismos que son empleados tanto como herramienta de trabajo, como de contacto con familiares y amigos. En otras palabras, se trata de una tecnología altamente integrada a las rutinas de este grupo de usuarios, a través de la cual trabajan y socializan.

Los temas que son seguidos por quienes dijeron trabajar en los medios, parecen confirmar lo anterior, con notables coincidencias entre Facebook y Twitter. Asuntos relacionados con el trabajo y temas que tienen que ver con noticias y asuntos de actualidad, son los que mayor frecuencia presentan. En un contexto como el de la región sureste de Coahuila ${ }^{15}$, destacan en lo particular asuntos que tienen que ver con la seguridad pública, como tema de seguimiento constante a través de Twitter.

Las opiniones expresadas por los editores de los periódicos que fueron entrevistados, permiten identificar algunas tendencias generales observadas en los antecedentes consultados. Tal como Spencer (2007) ha planteado, para los profesionales de la información noticiosa, Twitter permite tener acceso a sujetos que se encuentran cerca de los hechos noticiosos, sobre todo testigos de primera mano. Para este grupo de editores, además de esta posibilidad, Twitter se convierte en un espacio a través del cual es posible sondear los temas y asuntos que están siendo discutidos por sus usuarios, y que en muchas ocasiones, tienen un carácter noticioso.

La relativamente baja credibilidad que los sujetos participantes otorgan a la información que circula a través de Twitter, conduce a prácticas como las señaladas por Overholser (2009) y que en el caso analizado, se expresan en la confirmación de la información a partir de fuentes oficiales dentro de la misma plataforma, sobre todo en casos que tienen que ver con hecho violentos ocurridos en la localidad. Las cuentas en Twitter de los organismos públicos de seguridad pública se convierten en un referente obligado, son un indicador clave en el proceso de deslinde de rumores. En este mismo sentido, un aspecto que resulta oportuno destacar es que los editores recurran también a las cuentas de colegas periodistas para verificar la información obtenida a través de este medio. Hay, por lo tanto una

15 Desde febrero de 2010, en la región se han presentado diversos hechos violentos, y entre los que han destacado los que han tenido lugar en avenidas y calles céntricas de la ciudad de Saltillo, a partir de enfrentamientos entre diversos grupos de la delincuencia organizada, con las fuerzas del orden civiles y militares. 
discriminación de las fuentes en Twitter, y no todos los tweets reciben el mismo tratamiento. El reconocimiento y la validación entre pares resulta entonces en un relevante mecanismo de manejo de la información, al menos entre esta muestra de trabajadores en medios.

De manera coincidente por lo observado por Lariscya et al (2009), los editores de la muestra no recurren de manera sistemática a Twitter como única fuente para la generación de notas. Como ha sido mencionado, cuando algún asunto emergente irrumpe en la agenda, y no hay confirmación oficial oportuna, si la nota tiene la suficiente relevancia, se recurre al envío de un reportero para confirmar y profundizar en la información. Desde una perspectiva normativa, esto parece reforzar los mecanismos de validación de la información a los que están sujetos los emisores de información noticiosa. Recurriendo a la terminología de la sociocibernética, podríamos decir que los altos niveles de entropía, llevan a la ejecución de operaciones que la reduzcan, entre las que destaca la verificación a través de personas especialmente entrenadas para ello y que, al menos en teoría, son capaces de realizar acercamientos más o menos objetivos y profesionales a dichos eventos; bajo una estructura que se presume coherente con los criterios periodísticos a los que estén sujetos.

No obstante lo anterior, como Levy (2010) señala, el uso de Twitter está adquiriendo una mayor importancia y es empleado por un porcentaje significativo de trabajadores en los medios, tanto reporteros como editores. Como hemos señalado, se trata de un entorno en el que hay un flujo de información que los profesionales de las empresas informativas no pueden darse el lujo de ignorar, en buena parte debido a la velocidad con la que actualmente se actualiza y renueva la agenda. Ante el surgimiento y colocación destacada de una nota, pueden buscarse posteriormente mecanismos de verificación como los que ya han sido mencionados. Resulta evidente que los editores y profesionales de la noticia, no pueden permanecer al margen de este flujo de información.

Quizá uno de los aspectos que consideramos más relevantes a partir de los datos obtenidos en este trabajo, es que la esencia de las rutinas laborales dentro del proceso del newsmaking, no se ha transformado tanto como pudiera pensarse. Los valores fundamentales del periodismo como actividad profesional parecen reforzarse, y en todo caso, deben ajustarse a una agenda que incorpora a Twitter como un elemento entre muchos otros en su construcción. Los actores sociales que tradicionalmente influyen en este 
sentido, no parecen haber mutado de manera significativa. Si acaso, algunos de ellos, especialmente cuando se les demanda una rápida respuesta ante algún acontecimiento; se suman a Twitter como nodos con una altísima relevancia para los editores y reporteros. Se trata en general, de un contexto en el que la velocidad y las mediaciones que responden a ella, se ubican como herramientas pertinentes en la construcción social de la noticia.

\section{Bibliografía}

Cloutier, J. (1973): La communication audio-scripto-visuelle à l'heure des sefl-média. Montreal: Les Presses de l'Université de Montréal.

HermidA, A. (2010): "From TV to Twitter: How Ambient News Became Ambient Journalism", M/C Journal of Media and Culture, vol. 13, núm. 2, [documento electrónico]

http://journal.mediaculture.org.au/index.php/mcjournal/article/viewArticle/220.

ISLAS, O. (2012): "Sobre Twitter y la tuitósfera". El Universal Online. [documento electrónico], http://www.eluniversal.com.mx/articulos/69834.html

InTERnEt World Stats (2012): "Internet Usage Statistics for the Americas". [documento electrónico] http://www.internetworldstats.com/stats2.htm.

JEnkins, H. (2006): Convergence Culture: Where Old and New Media Collide. Nueva York: New York University Press.

Jürgens, P., Jungherr, A. y Schoen, H. (2011): "Small Worlds with a Difference: New Gatekeepers and the Filtering of Political Information on Twitter". pp. 1-5. Proceedings of the ACM WebSci'1 1, pp 1-5, Alemania.

Kongsved, S. M.; Basnov, M.; Holm-Christensen y K. Huollund, N. H. (2007): "Response Rate and Completeness of Questionnaires: A Randomized Study of Internet Versus Paper-and-Pencil Versions", Journal of Medical Internet Research, 9 (3).

Landow, G. (1994) (Ed): Hyper / Text / Theory. Baltimore: Johns Hopkins University Press.

Lariscya, R. W., Averyb, E. J., Sweetser, K. D. y Howes, P. (2009): "An examination of the role of online social media in journalists' source mix", Public Relations Revier, 35, pp. 314-316.

Mediaciones Sociales, № 12, 2013, pp. 108-128. ISSN electrónico: 1989-0494.

DOI: http://dx.doi.org/10.5209/rev_MESO.2013.n12.45265 
LEVY, G. (2010): Social Media and Journalism: How the Internet and Social Networking Have Changed Journalism's Workflow. Tesis de posgrado. Boston: Tufts University.

LÈvy, P. (1997): Collective Intelligence. EEUU: Perseus Books.

MaIGRet, É. (2005): Sociología de la comunicación y de los medios. México: Fondo de Cultura Económica.

Meneses, M.E. (2011). Periodismo convergente. Tecnología, medios y periodistas en el Siglo XXI. México: Porrúa.

Overholser, G. (2009): " What Is Journalism’s Place in Social Media?", Nieman Reports, otoño de 2009, [documento electrónico] http://www.nieman.harvard.edu/reports/article/101882/What-Is-JournalismsPlace-in-Social-Media.aspx

Shoemaker, P. y Reese, S. (1994): La mediatización del mensaje. México: Diana.

SPEnCER, J. (2007): "Found in (My)Space: Social networking sites like MySpace and Facebook are valuable sources of information for journalists", American Journalism Review, vol.29; número 5, pp. 36-39.

Wolf, M. (2005): La investigación de la comunicación de masas. Crítica y perspectivas. México: Paidós. 


\section{PARA CITAR ESTE TRABAJO EN BIBLIOGRAFÍAS:}

PÉREZ SALAZAR, Gabriel, CARABAZA GONZÁLEZ, Julieta I. y ROMO GUTIÉRREZ, Daniel O. (2013): "El uso de Twitter en las salas de redacción: redes sociales y newsmaking en la región sureste de Coahuila, México", Mediaciones Sociales. Revista de Ciencias Sociales y de la Comunicación, $\mathrm{n}^{\circ}$ 12, pp. 108-128. DOI: http://dx.doi.org/10.5209/rev_MESO.2013.n12.45265

\section{Los autores}

${ }^{(*)}$ Gabriel Pérez Salazar es doctor en Ciencias Políticas y Sociales, con orientación en Comunicación por la Universidad Nacional Autónoma de México. Profesor de tiempo completo en la Facultad de Ciencias de la Comunicación de la Universidad Autónoma de Coahuila, Unidad Saltillo, México. Coordinador del Grupo de Investigación "Internet, Nuevas Tecnologías y Sociedad de la Información" de la Asociación Mexicana de Investigadores de la Comunicación (AMIC). Miembro del Sistema Nacional de Investigadores del Consejo Nacional de Ciencia y Tecnología (CONACYT), nivel I.

${ }^{(* *)}$ Julieta I. Carabaza González es doctora en Comunicación Social por la Universidad de la Habana, Cuba. Profesora de Tiempo Completo de la Facultad de Ciencias de la Comunicación, de la Universidad Autónoma de Coahuila, Unidad Saltillo. Miembro del Sistema Nacional de Investigadores del Consejo Nacional de Ciencia y Tecnología (CONACYT), nivel I.

${ }^{(* * *)}$ Daniel Osvaldo Romo Gutiérrez es alumno tesista de licenciatura en la Facultad de Ciencias de la Comunicación, de la Universidad Autónoma de Coahuila, Unidad Saltillo, con el trabajo "La red social Twitter como fuente de información periodística. El caso de los editores en los periódicos Vanguardia, Zócalo Saltillo y El Diario de Coahuila”, bajo la dirección del Dr. Gabriel Pérez Salazar.

RECIBIDO: 10 de enero de 2013.

ACEPTADO: 25 de junio de 2013.

Mediaciones Sociales, № 12, 2013, pp. 108-128. ISSN electrónico: 1989-0494. DOI: http://dx.doi.org/10.5209/rev_MESO.2013.n12.45265 